\title{
Computerized Tomography with High-Energy Proton Beams: Tomographic Image Reconstruction from Computer-Simulated Data
}

\author{
Ivan Evseev ${ }^{1}$, Margio C. L. Klock ${ }^{1}$, Sergei A. Paschuk ${ }^{1}$, Hugo R. Schelin ${ }^{1}$, João A. P. Setti ${ }^{1}$, \\ Ricardo T. Lopes ${ }^{2}$, Reinhard W. Schulte ${ }^{3}$, and David C. Williams ${ }^{4}$ \\ ${ }^{1}$ Federal Center of Technological Education - CEFET/PR, Curitiba - PR - Brazil. \\ ${ }^{2}$ Nuclear Instruments Laboratory - LIN/UFRJ, Rio de Janeiro - RJ - Brazil. \\ ${ }^{3}$ Loma Linda University Medical Center, Loma Linda - CA - USA. \\ ${ }^{4}$ Santa Cruz Institute of Particle Physics, University of California, Santa Cruz - CA - USA.
}

Received on 18 September, 2003

\begin{abstract}
The use of protons instead of $X$-rays for computerized tomography (CT) studies has potential advantages, especially for medical applications in proton treatment planning. However, the spatial resolution of proton CT is limited by multiple Coulomb scattering (MCS). We used the Monte Carlo simulation tool GEANT4 to study the resolution achievable with different experimental arrangements of a proton CT scanner. The passage of a parallel $200 \mathrm{MeV}$ proton beam through a virtual cylindrical aluminum phantom with $50 \mathrm{~mm}$ external diameter was simulated. In our study, the phantom contained a set of cylindrical holes with diameters ranging from $4 \mathrm{~mm}$ to $0.5 \mathrm{~mm}$. The GEANT4 simulation consisted of a series of 180 projections at 2 degree intervals with 350 proton track histories for each one. The filtered back projection algorithm was used to reconstruct a $2 D$ tomographic image of phantom.
\end{abstract}

\section{Introduction}

Proton radiation therapy is a highly precise form of cancer therapy, which spares more healthy tissue and allows higher tumor doses than conventional radiation therapy. This is possible due to the characteristic of the proton depth dose curve: a relatively low entrance dose is followed by a highdose Bragg peak, which can be positioned in the tumor tissue. Beyond the Bragg peak the dose fall-off is very steep, i.e., from $90 \%$ to $20 \%$ of the peak dose within a few millimeters. Precise and conformal radiation therapy with protons therefore requires a very accurate prediction of the position of the Bragg peak within the patient to avoid damage to normal tissues.

In existing proton treatment centers, dose calculations are performed based on $X$-ray computerized tomography (CT) and the patient is positioned with $X$-ray radiographs. The use of $X$-ray CT images for proton treatment planning is inherently inaccurate, because it ignores fundamental differences in physical interaction processes between photons and protons. Further, $X$-ray radiographs depict well only skeleton structures; frequently they do not have enough contrast to show the tumor itself. The use of charged particle beams for imaging, as an alternative, shows an excellent density resolution [1],[2]. Ideally, one would image the tumor directly with proton $\mathrm{CT}$ ( $\mathrm{pCT}$ ) by measuring the energy loss of high-energy protons that traverse the patient.

Although the idea of pCT is not new, and some previ- ous experimental work has been published [3],[4],[5], pCT is currently not available. In the past 3 decades, the forces of scientists and engineers were concentrating on the progress in conventional $X$-ray CT, mainly because of economical reasons. With the development of medical proton gantries, first at Loma Linda University Medical Center, and now in several other proton treatment centers, progress in multichannel detector systems, like the silicon strip detectors (SSD), and the orders-of-magnitude increase in computing power and speed, technical and, consequently, economical obstacles for the development of pCT have been overcome.

However, due to the physical nature of the process, pCT has some limitations. For example, the spatial resolution of the method is limited by multiple Coulomb scattering (MCS) of the protons in the investigated sample. We used Monte Carlo simulations to study the spatial resolution achievable with different experimental schemes of a proton CT scanner.

\section{Simulation of projections}

The passage of a parallel proton beam with $E_{i n}=200 \mathrm{MeV}$ through a virtual cylindrical aluminum $\left(\rho=2.7 \mathrm{~g} / \mathrm{cm}^{3}\right)$ phantom was simulated using the GEANT4 software [6]. Fig.1 shows the geometry of simulated experimental setup. 


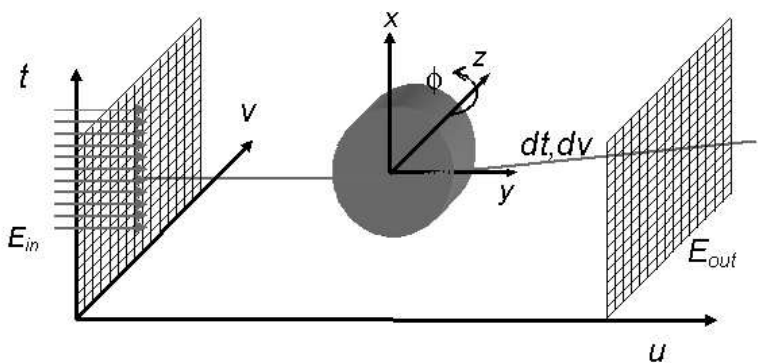

Figure 1. The setup of simulated experiment.

The beam reference system $(t, u, v)$ was considered stationary, while the local phantom coordinate system $(x, y, z)$ was systematically rotated about the $z$-axis. The initial proton beam had a width of $70.0 \mathrm{~mm}$ along the $t$-coordinate, zero width in the $v$-coordinate direction, and was centered at $t=v=35.0 \mathrm{~mm}$. The protons were propagated in the direction of the $u$-coordinate.

The aluminum phantom, similar to that one used in [7], had $50 \mathrm{~mm}$ external diameter and a set of cylindrical holes with diameters ranging from $4 \mathrm{~mm}$ to $0.5 \mathrm{~mm}$ (see Table 1). It was situated at $t=35.0 \mathrm{~mm}$ and $u=150.0 \mathrm{~mm}$ (central point) like it is shown by Fig. 2 .

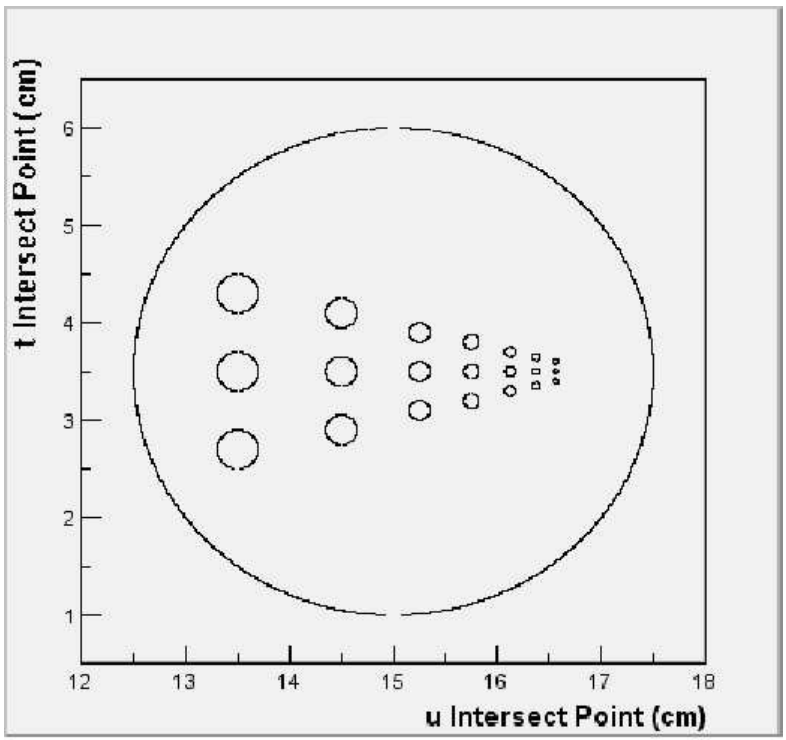

Figure 2. The phantom geometry.

The characteristics of outgoing protons for a given angle of the phantom rotation (projection) were determined at the $t v$-plane, symmetrically situated at $u=30.0 \mathrm{~mm}$. The interaction of protons with the air in the phantom holes and on the way to and behind the phantom was neglected. The energy of each outgoing proton $E_{\text {out }}$, and its new $t$ - and $v$ position were the principal characteristics contained in the program output file. More technical details of the simulation can be found in [8].
TABLE 1 . The phantom geometry.

\begin{tabular}{|c|c|c|c|}
\hline Structure & $\mathbf{D}[\mathbf{m m}]$ & $\mathbf{x}_{0}[\mathbf{m m}]$ & $\mathbf{y}_{0}[\mathbf{m m}]$ \\
\hline hole 1a & 4.0 & -15.0 & 8.0 \\
\hline hole 1b & 4.0 & -15.0 & 0.0 \\
\hline hole 1c & 4.0 & -15.0 & -8.0 \\
\hline hole 2a & 3.0 & -5.0 & 6.0 \\
\hline hole 2b & 3.0 & -5.0 & 0.0 \\
\hline hole 2c & 3.0 & -5.0 & -6.0 \\
\hline hole 3a & 2.0 & 2.5 & 4.0 \\
\hline hole 3b & 2.0 & 2.5 & 0.0 \\
\hline hole 3c & 2.0 & 2.5 & -4.0 \\
\hline hole 4a & 1.5 & 7.5 & 3.0 \\
\hline hole 4b & 1.5 & 7.5 & 0.0 \\
\hline hole 4c & 1.5 & 7.5 & -3.0 \\
\hline hole 5a & 1.0 & 11.25 & 2.0 \\
\hline hole 5b & 1.0 & 11.25 & 0.0 \\
\hline hole 5c & 1.0 & 11.25 & -2.0 \\
\hline hole 6a & 0.75 & 13.75 & 1.5 \\
\hline hole 6b & 0.75 & 13.75 & 0.0 \\
\hline hole 6c & 0.75 & 13.75 & -1.5 \\
\hline hole 7a & 0.5 & 15.625 & 1.0 \\
\hline hole 7b & 0.5 & 15.625 & 0.0 \\
\hline hole 7a & 0.5 & 15.625 & -1.0 \\
\hline
\end{tabular}

The simulation reported here consisted of a series of 180 projections at 2-degree intervals with 350 proton track histories for each projection. In spite of the fact that the $180^{\circ}$ rotation is enough for tomographic image reconstruction, the simulation of the full $360^{\circ}$ rotation of the sample was done to check the reproducibility of the results.

\section{Tomographic image reconstruction}

To reconstruct the $2 D$ tomographic image of the phantom from the GEANT4 simulated data, we used the so-called "filtered back projection method" [9]. This method is commonly used in conventional $X$-ray CT. In the latter case, the intensity of the $X$-ray beam that follows a straight pass of length $L$ in through a medium is given by:

$$
I\left(E_{\gamma}\right)=I_{0}\left(E_{\gamma}\right) \cdot \exp \left(-\int_{0}^{L} \mu\left(x, E_{\gamma}\right) \cdot d x\right)
$$

where $\mu\left(x, E_{\gamma}\right)$ describes the distribution of the linear coefficient of photon absorption along the path. It should be stressed, that the absorption depends not only on the material of the absorber, but also on the $X$-ray energy $E_{\gamma}$. The energy spectrum of an $X$-ray tube, $I_{0}\left(E_{\gamma}\right)$, is an empirically determined complex function of the tube construction and regime of operation.

The "signal", which is back projected by the method to achieve a tomographic image, is given by: 


$$
S_{\gamma}(t, \varphi)=\ln \left(\frac{N_{0}}{N(t, \varphi)}\right)=\mu_{\text {measured }} \cdot L
$$

where $N_{0}$ and $N(t, \varphi)$ are the experimentally registered initial $x$-ray beam intensity and outgoing beam intensity for the given beam position $t$ and the angle of CT scanner rotation $\varphi$, respectively. (For simplicity, we here assumed the so-called "1st generation" CT scanner scheme [9].)

In the case of a proton beam, the energy loss can be calculated using the expression [4]:

$$
\Delta E=E_{0}-E=\int_{0}^{L} \mu_{S P}\left(x, E_{p}\right) \cdot d x
$$

where we used the non-traditional expression $\mu_{S P}\left(x, E_{p}\right)$ instead of the commonly used $d E / d x$ for the local stopping power, just to stress the existing analogy in the equations. The stopping power depends not only on the material, but is also a strong function of the local proton energy $E_{p}$. The proton energy will significantly change along the path through a thick sample. Thus, when we assume that the initial proton beam energy is maintained along the path, the situation is similar to the case of polychromatic $X$-rays. The backprojected signal may then be defined as:

$$
S_{p}(t, \varphi)=\Delta E(t, \varphi)=E_{0}-E(t, \varphi)=\mu_{S P \text { measured }} \cdot L
$$

The similarity of expressions (2) and (4) was a strong motivation to use of the same method for the pCT image reconstruction as for the $X$-ray CT reconstruction. Of course, one can expect the same problem - the presence of the socalled "polychromaticity artifacts" [9] - in the reconstructed images.

\section{Results and discussion}

As expected, our results were strongly affected by polychromatic artifacts (see Fig.3 and Fig.4). However, the aim of current investigation was to determine the spatial resolution of the reconstruction method; therefore, we will not further discuss these artifacts, which will have to be resolved if $\mathrm{pCT}$ is used for radiotherapy treatment planning [10].

The idea of image reconstruction is based on Nyquist's theorem, i.e., our signal (projection) should be "sampled" at equal intervals (with a given spatial frequency) [9]. In a real $X$-ray CT scanner, this is always true, due to its construction (equal translation motion step for the first generation CT scanners, or regular $X$-ray detector cell structure for the last generations).

However, the structure of the simulated proton data is different. The initial $t$-coordinate of a proton along the width of the parallel beam (see Fig.1) is a random number with uniform distribution between $0.0 \mathrm{~mm}$ and $70.0 \mathrm{~mm}$. From the experimental point of view, this corresponds to the use of a coordinate sensitive detector with an infinite spatial resolution. The same is true for the outgoing proton $t$ coordinate. (For simplicity, we ignored the proton scattering in $v$-direction during the current analysis, having in mind the use of a one-sided SSD for $t$-coordinate determination for the incoming and outgoing protons in a real experiment.)

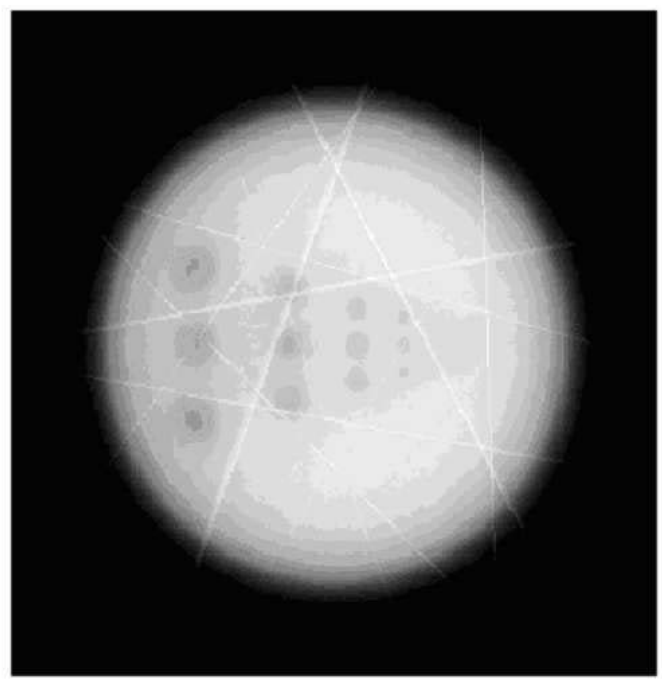

Figure 3. The result of filtered back projection reconstruction of the proton energy loss data as a function of initial proton position.

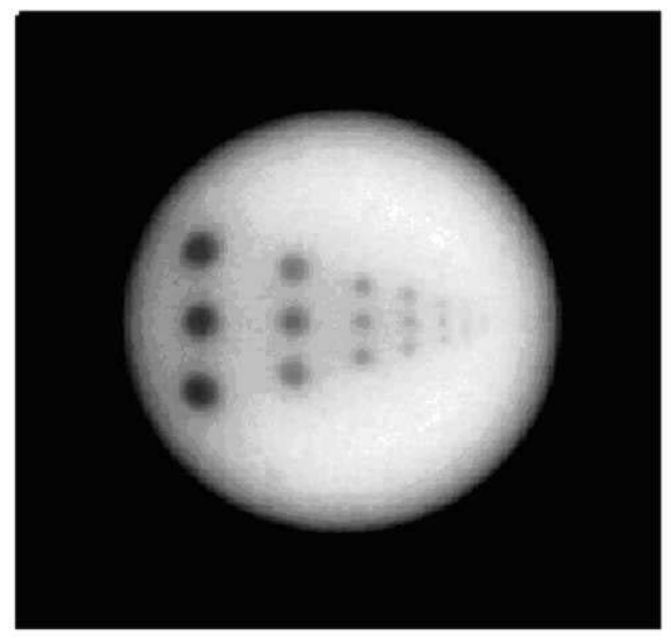

Figure 4. The phantom pCT image after data correction assuming straight line approximation.

Thus, before the pCT image reconstruction, the initial and outgoing $t$-values for each proton in all projections should be rounded to the nearest center of sampling interval (hypothetical strip position). A $0.2 \mathrm{~mm}$ strip pitch was assumed for a virtual (but quite realistic) SSD, and, consequently, one can expect a $0.4 \mathrm{~mm}$ limit of resolution for the reconstructed CT image. A better resolution, although technically possible, is not desirable in our case because under our given conditions ( 350 protons per projection of $70.0 \mathrm{~mm}$ width) this would lead to "empty rows" in the data matrix.

In line with the comments above, the pCT images were reconstructed using an "ideal" filter [9] on the $350 \times 350$ 
pixel matrix on a $0-255$ gray scale. Using only the initial $t$-coordinate versus $\Delta E$ data leads to the image shown in Fig.3. From the experimental point of view, this corresponds to a simple experimental scheme with a single SSD in front of the object, and a proton energy detector without position sensitivity behind the object. With this scheme, only the phantom holes of $1.5 \mathrm{~mm}$ diameter and larger are visible. The presence of strong polychromatic artifacts is also obvious.

An improved pCT image with reasonable contrast and spatial resolution (see Fig.4) was reached when both initial and final coordinates were used to reconstruct a straightline path of the protons through the phantom. In this case, phantom holes of $1 \mathrm{~mm}$ diameter can clearly be seen, while the location of smaller holes $(0.75 \mathrm{~mm}$ and $0.5 \mathrm{~mm})$ can be guessed. The image illustrates the value of correcting the effect of the MCS.

\section{Conclusions}

A moderate spatial resolution can be achieved by using a position-sensitive proton detector in front of the object, and a proton energy detector without position sensitivity behind the object. If both entrance and exit position are taken into account to determine a straight-path approximation, a much improved resolution can be achieved. Thus, an experimental scheme with two position-sensitive detectors, one situated in the front and the other behind the object, should lead to a reasonably high spatial resolution. Further improvement of the spatial resolution can be expected when using reconstruction techniques that allow incorporation of models of proton MCS better than the straight-line approximation.

\section{Acknowledgments}

The authors are very thankful to the Brazilian agencies
CNPq, CAPES and Fundação Araucária for financial support.

R.W.Schulte's research was sponsored by the U.S. Dept of Army (C/A \# DAMD17 - 97 - 2-7016) and the National Medical Technology Testbed (NMTB), and the content and information does not reflect the position or policy of the U.S. government, or NMTB; no official endorsement should be inferred.

\section{References}

[1] E.V. Benton, R.P. Henke, and C.A. Tobias, Science 182, 474 (1973).

[2] B.A. de Souza, S.C. Cabral, and R.T. Lopes, Radiation Measurements 24, 187 (1995).

[3] A.M. Cormack and A.M. Koehler, Phys. Med. Biol. 21, 560 (1976).

[4] K.M. Hanson, J.N. Bradbury, T.M. Cannon et.al., Phys. Med. Biol. 26, 965 (1981).

[5] K.M. Hanson, J.N. Bradbury, R.A. Koeppe, et.al., Phys. Med. Biol. 27, 25 (1982).

[6] GEANT 4 Home Page: http://wwwinfo.cern.ch/asd/geant4/geant $4 . h t m l$

[7] T. Taylor and L.R. Lupton - Resolution, Artifacts and Design of Computed Tomography Systems - Nuclear Instruments and Methods in Physics Research, A242, 603 (1986).

[8] Home Page: http://scipp.ucsc.edu/ davidw/radiobiology/scan2/.

[9] A.C. Kak and M. Slaney, Principles of Computerized Tomographic Imaging, IEEE Press Inc., N.Y., USA, 1988.

[10] U. Schneider, E. Pedroni, and A. Lomax, Phys. Med. Biol. 41, 111 (1996). 